\title{
Bedside echocardiography for diagnosis of Intracardiac cement embolism after percutaneous vertebroplasty: A case report
}

\author{
Panpan Yin ${ }^{1}$, Junli $\mathrm{Hu}^{1}$, Shaochun Wang ${ }^{1}$, Guiling Sui ${ }^{1}$, Guozhen Yuan ${ }^{1}$, and Dongchen \\ $\mathrm{FAN}^{1}$
}

${ }^{1}$ Affiliation not available

March 21, 2021

\begin{abstract}
Abstract The purpose of this paper is to report a case diagnosed by bedside echocardiography in which bone cement infiltrated into the paravertebral vein system after percutaneous vertebroplasty (PVP) and caused intracardiac cement embolism (ICE). A 79-year-old female patient had suddenly become unconscious 14 hours after PVP. Emergency bedside echocardiogram showed that the patient had a strong echo in the right heart with a small amount of pericardial effusion, suspected of causing cardiogenic shock. Computed tomography (CT) showed high density in the distal branches of both pulmonary arteries and a high density in the right heart.Combined with the history of surgery, the clinician considers the foreign body as bone cement and the diagnosis was ICE. The bone cement in the heart was removed under emergency cardiopulmonary bypass. The patient recovered and was discharged smoothly.
\end{abstract}

\section{Title:}

Bedside echocardiography for diagnosis of Intracardiac cement embolism after percutaneous vertebroplasty: A case report

Running title: Diagnosis of cement embolism after PVP

Authors: Panpan Yin ${ }^{1}$, Junli $\mathrm{Hu}^{1}$, ShaochunWang ${ }^{1}$, Guiling Sui ${ }^{1}$, Guozhen Yuan ${ }^{1}$, Dongchen Fan ${ }^{1}$

Affiliations: Full information for each author

${ }^{1}$ Department of Ultrasound, the Affiliated Hospital of Jining Medical University, Shandong Jining, China

\section{Address for Correspondence :}

Dongchen Fan, BS

Department of Ultrasound, The Affiliated Hospital of Jining Medical University

No. 89, Guhuai Road, Rencheng District, Shandong Jining, China

Email: fandongchenvip@163.com

ORCID iDs :

Dongchen Fan https://orcid.org/0000-0002-1015-536X

Panpan Yin https://orcid.org/0000-0003-2048-6346

Junli Hu https://orcid.org/0000-0002-7111-3702

ShaochunWang https://orcid.org/0000-0002-7392-5077 
Guiling Sui https://orcid.org/0000-0003-3091-3567

Guozhen Yuan https://orcid.org/0000-0003-2068-799X

Bedside echocardiography for diagnosis of Intracardiac cement embolism after percutaneous vertebroplasty: A case report

\section{Abstract}

The purpose of this paper is to report a case diagnosed by bedside echocardiography in which bone cement infiltrated into the paravertebral vein system after percutaneous vertebroplasty (PVP) and caused intracardiac cement embolism (ICE). A 79-year-old female patient had suddenly become unconscious 14 hours after PVP. Emergency bedside echocardiogram showed that the patient had a strong echo in the right heart with a small amount of pericardial effusion, suspected of causing cardiogenic shock. Computed tomography (CT) showed high density in the distal branches of both pulmonary arteries and a high density in the right heart.Combined with the history of surgery, the clinician considers the foreign body as bone cement and the diagnosis was ICE. The bone cement in the heart was removed under emergency cardiopulmonary bypass. The patient recovered and was discharged smoothly.

\section{Keywords}

Bedside echocardiographic, Bone cements, Cardiac embolism, Percutaneous vertebroplasty

\section{A Data Availability Statement}

The data that support the findings of this study are available from the corresponding author upon reasonable request.

Funding: None

Conflict of Interest: The authors declare that there are no conflict of interests.

Patient consent statement: Informed consent of patient was obtained for the study.

\section{Author Contributions}

Concept/design: Dongchen Fan, ShaochunWang. Analysis/interpretation: Guiling Sui, Junli Hu.

Drafting article: Panpan Yin. Critical revision of article: Dongchen Fan, ShaochunWang.

Other: Panpan Yin, Guozhen Yuan.

\section{INTRODUCTION}

PVP is a minimally invasive surgery to treat osteoporotic vertebral compression fractures by injecting bone cement into the diseased vertebral body. ${ }^{1}$ It can effectively restore the stability of the vertebral body and quickly relieve pain, but it has many complications, especially bone cement leakage. ${ }^{2-3}$ Studies have shown that the incidence of bone cement leakage into the heart is $3.9 \%,{ }^{4}$ which are associated with a higher risk of death. Timely and accurate detection of pulmonary embolism or ICE is of great value for early and effective treatment. However, patients with pulmonary embolism or ICE are often bedridden, it is difficult to move to receive various imaging tests. Echocardiography is non-invasive, simple and unique for detecting solid bodies, and can detect myocardial injury and pericardial effusion caused by intracardiac foreign bodies. This paper reports a case of bone cement cardiac embolism and cardiac perforation after PVP which is diagnosed by bedside echocardiography.

\section{CASE DESCRIPTION}

A 79-year-old female patient was diagnosed with osteoporotic vertebral compression fracture and decided to undergo PVP surgery. Preoperative echocardiography showed that the left ventricular ejection fraction was $62 \%$, the ascending aorta was dilated (which may be related to the patient's long-term hypertension), and the left ventricular diastolic function was reduced. However, 14 hours after the operation, the patient suddenly 
became unconscious after moving, vomited a small number of stomach contents, urinated incontinence, and unable to move his limbs. The doctor immediately gave oxygen inhalation and intravenous infusion. Then the patient gradually became conscious and able to move his limbs, but his blood pressure was unstable, with a minimum of $55 / 38 \mathrm{mmHg}$. Emergency bedside echocardiography showed that the left ventricular ejection fraction was $53 \%$, and the ventricular systolic function was acceptable. A strong U-shaped echo with a length of about $6.5 \mathrm{~cm}$ can be seen in the right ventricle, which is stored in the right ventricle across the tricuspid valve (Fig. 1.A). A moderate regurgitation signal can be seen in the tricuspid valve, and the pericardial effusion with a depth of about $10 \mathrm{~mm}$ can be seen in the pericardial cavity, the sound transmission is poor. Combined with the history of PVP, the doctor suspected that the patient might be a cardiogenic shock caused by the bone cement in the heart. CT showed high density in the vertebral venous plexus and the odd veins on the left side of the thoracic spine, and there were multiple dots and high-density shadows in the distal branches of both pulmonary arteries. It also indicated a high density in the right atrium and hemopericardium (Fig. 2).

After the cardiac surgery consultation, the foreign body removal operation under cardiopulmonary bypass was urgently performed. During the operation, a block of U-shaped bone cement was found in the right atrium (Fig. 3). The tip penetrated into the right ventricular wall through the right ventricular side of the anterior valve of the tricuspid valve, and the caudal end was located in the right atrium. The bone cement was taken out and sutured to repair the wall of the right ventricle. Exploration revealed that part of the chordae of the posterior leaflet of the tricuspid valve was ruptured, so a tricuspid valve repair was performed. The patient was discharged smoothly after 25 days of recovery. The echocardiogram before discharge showed mild tricuspid regurgitation and mitral regurgitation, the pericardial effusion was not detected (Fig. 1. B and C). One month later, the echocardiography was rechecked, and there were no obvious other abnormalities.

\section{DISCUSSION}

In this case, the patient suffered a sudden loss of consciousness about 14 hours after PVP. Emergency bedside echocardiography showed U-shaped strong echoes in the right heart and pericardial effusion in the apex of the heart. Combined with the operation history and CT, it was considered that the leaking cement entered the lumbar segment vein through the paravertebral plexus, and entered the superior vena cava and the right atrium through the odd vein. Eventually, it entered the superior vena cava and the right atrium through the odd vein, and the bone cement gradually hardened and bended as it traveled with the blood flow, so that the tip pierced the heart and caused perforation of the heart. This is the first reported case of a patient who suffered a sudden loss of consciousness after PVP within one day, and the doctor made the diagnosis of ICE in a very short time.

Previous studies have found that most patients with ICE have dyspnea or chest pain as the first symptoms after PVP, ${ }^{5-8}$ the duration of onset is inconsistent, and rare cases may be asymptomatic. ${ }^{6}$ But no matter what the clinical symptoms are, the most urgent need is for early detection and immediate treatment. Xray has special advantages for the discovery of metallic foreign bodies, but it can usually not determine the specific location of the mass in the heart cavity, and some chest X-rays only show thickening of lung texture. ${ }^{8}$ Chest CT can confirm the location of cement emboli in the heart, and at the same time can judge whether there is pulmonary embolism, ${ }^{5,7-8}$ but it takes a long time for the examination and the results to be obtained, and it is impossible to observe the immediate changes of the foreign body.

The heart is a motor organ, the attention should be paid to the migratory nature of foreign bodies during diagnosis. Echocardiography has almost $100 \%$ sensitivity for assessing the size, position and mobility of foreign bodies in the heart. ${ }^{9}$ Bedside echocardiography is non-invasive and simple, especially for detecting non-metallic foreign bodies. It can identify valve damage, intracardiac shunt and detect myocardial tears caused by intracardiac foreign bodies. Because of similar clinical symptoms, ICE is most likely to be misdiagnosed as coronary atherosclerotic heart disease, and the computed tomography angiography sometimes show negative results, ${ }^{5}$ the bedside echocardiography is also helpful in distinguishing that primary heart diseases. 
This case reminds that when encountering patients with a sudden loss of consciousness or other atypical symptoms after PVP, it is necessary to perform echocardiography as soon as possible to assess the heart condition, which is consistent with the view of Yu Song. ${ }^{8}$ Special attention should be paid to the possibility of ICE, especially echocardiography shows a strong echo of a foreign body in the heart accompanied by pericardial effusion in patients after PVP.

\section{CONCLUSION}

Bedside echocardiography can accurately characterize and locate heart foreign bodies, Combined with the medical history and other related examinations, which can provide a strong reference for clinical treatment, so as to save precious time for saving patients' lives.

\section{REFERENCES}

1. Avram Allan Edidin, Kevin L.Ong, Edmund Lau, Steven M Kurtz. Morbidity and Mortality After Vertebral Fractures: Comparison of Vertebral Augmentation and Nonoperative Management in the Medicare Population.Spine (Phila Pa 1976) 2015;40(15):1228-41.

2. Wen-Jer Chen, Yu-Hsien Kao, Shih-Chieh Yang, Shang-Won Yu, Yuan-Kun Tu, Kao-Chi Chung. Impact of Cement Leakage Into Disks on the Development of Adjacent Vertebral Compression Fractures. J Spinal Disord Tech 2010;23(1):35-9.

3. Douglas J Martin, Arash Ehteshami Rad, David F Kallmes. Prevalence of extravertebral cement leakage after vertebroplasty: procedural documentation versus CT detection. Acta Radiol 2012;53(5):56972.

4. Sarah Fadili Hassani, Evelyne Cormier, Eimad Shotar, Mehdi Drir, Jean-Philippe Spano, Laetitia Morardet, eat. Intracardiac cement embolism during percutaneous vertebroplasty: incidence, risk factors and clinical management. Eur Radiol, 2019; 29(2): 663-673.

5. Patrick Swojanowsky, Maria Brinkmeier-Theofanopoulou, Claus Schmit, Uwe Mehlhorn. A rare cause of pericardial effusion due to intracardiac cement embolism. Eur Heart J, 2018; 39(32):3001.

6. Renee Duijvelshoff, Nathalie F M Anthonissen, Wim J Morshuis, Leen Van Garsse. Intracardiaccement embolism resulting in tricuspid regurgitation. Eur J Cardiothorac Surg, 2019; 55(2):366-8.

7. Tomás F Cianciulli, Diego E Mc Loughlin, Luis A Morita, María C Saccheri, Jorge A Lax. Bone cement cardiac and pulmonary embolism. Echocardiography, 2017; 34(8):1239-41.

8. Yu Song, Xiaofan Huang, Long Wu. Removal of intracardiac bone cement embolism after percutaneous kyphoplasty: A case report.Medicine, 2020, 99(11):19354.

9. YiSupomo, Handy Darmawan. An Unusual Foreign Body in the Heart: A Case Report. Ann Thorac Cardiovasc Surg, 2018; 24(4):205-7.

\section{Figure Legends}

Figure 1.
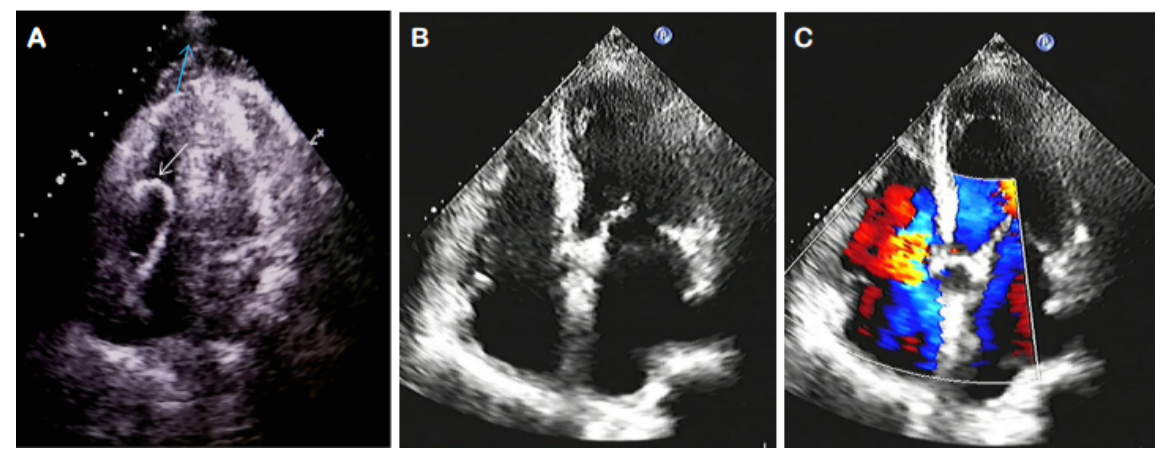

Fig.1. Ultrasound images of bone cement in bedside echocardiography before surgery and one month after 
surgery.(A) A strong echo in the right heart cavity (write arrow); A small amount of pericardial effusion (blue arrow). (B) Apical four-chamber heart without abnormal echo after surgery. (C)Mild tricuspid regurgitation and mitral regurgitation under color doppler after surgery.

\section{Figure 2.}
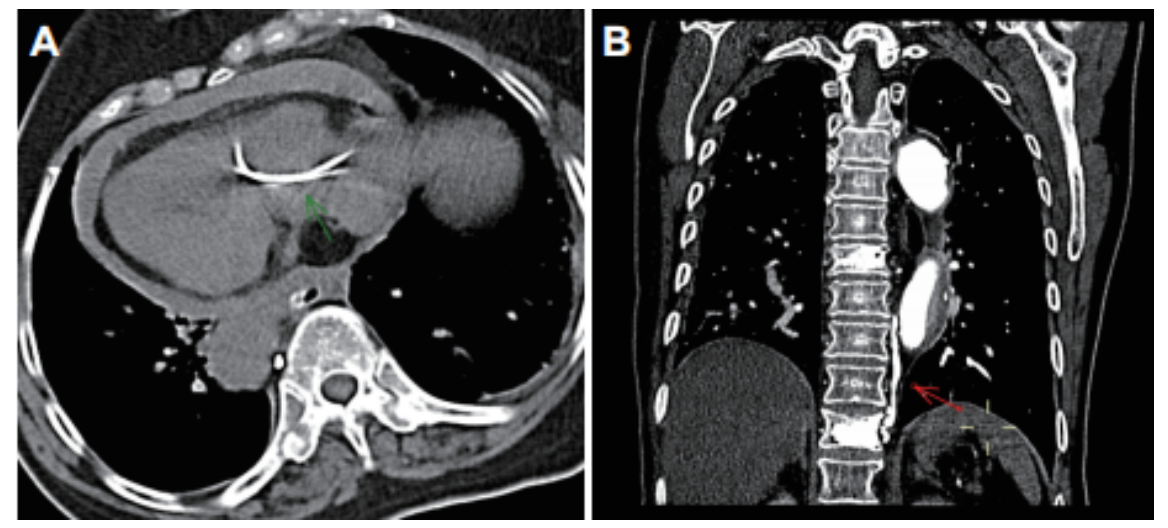

Fig.2. Chest computed tomography images of bone cement.(A) High density shadow in the right heart and hemopericardium.(B) High density shadow in the vertebral venous plexus and pulmonary arteries.

\section{Figure 3.}




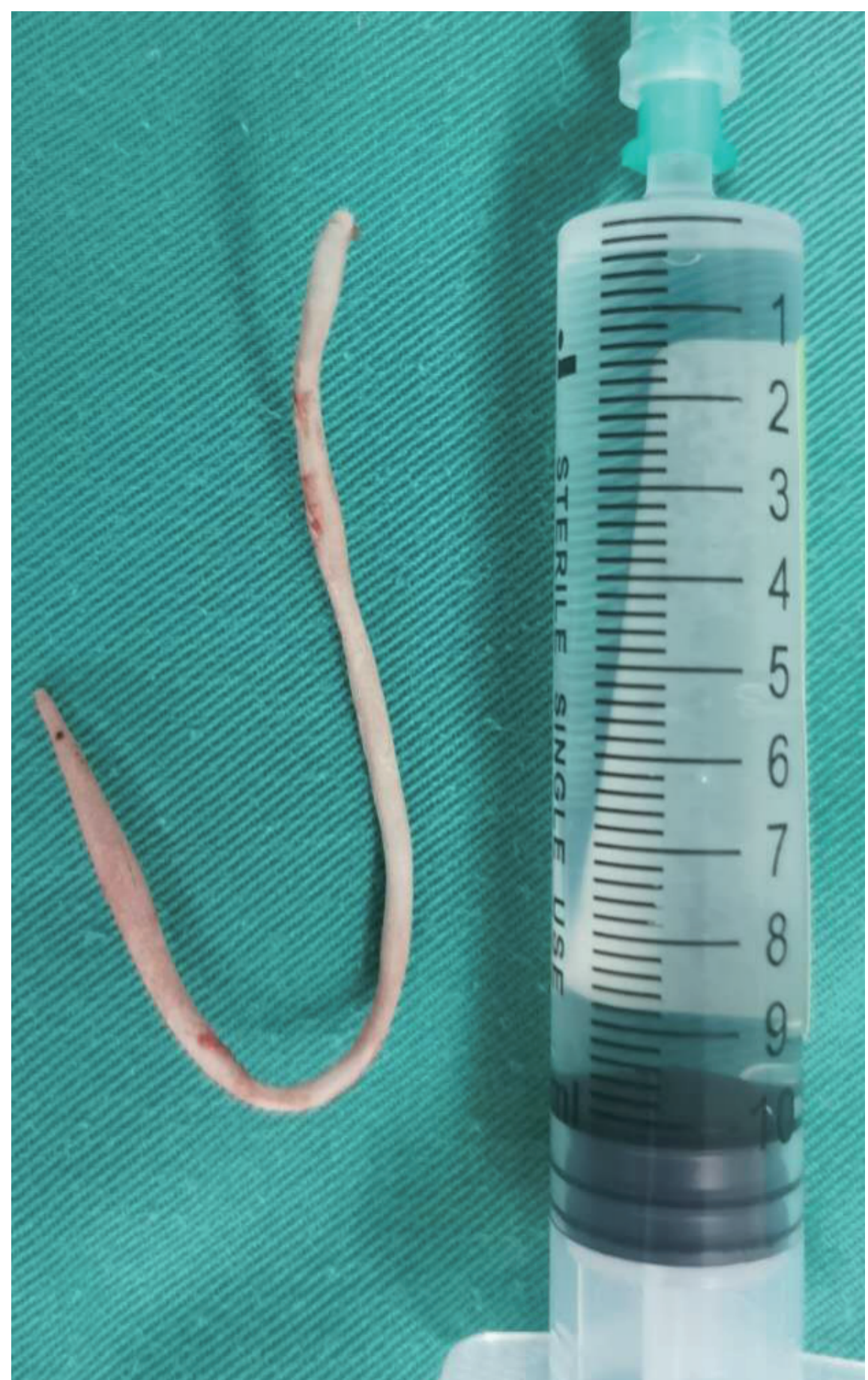

Fig.3. The removed bone cement from the right heart.

\section{Hosted file}

Figure.pdf available at https://authorea.com/users/403036/articles/514709-bedside- 
echocardiography-for-diagnosis-of-intracardiac-cement-embolism-after-percutaneousvertebroplasty-a-case-report 